\title{
ARTICLE
}

Received 7 Jun 2012 | Accepted 6 Jun 2013 | Published 1 Jul $2013 \quad$ DOl: 10.1038/ncomms3111

\section{Spatiotemporally controlled induction of autophagy-mediated lysosome turnover}

Yu-Hsien Hung ${ }^{1,2}$, Lily Man-Wen Chen ${ }^{1}$, Jin-Yi Yang ${ }^{1} \&$ Wei Yuan Yang, ${ }^{1,2}$

Lysosomes are the major degradative compartments within cells, harbouring a wide variety of hydrolytic enzymes within their lumen. Release of lysosomal hydrolases from lysosomes into the cell cytoplasm results in cell death. Here we report that damaged lysosomes undergo autophagic turnover. Using a light-induced lysosome impairing scheme that can be controlled spatially and temporally within a cell, we show that damaged lysosomes are selectively ubiquitinated, recruit autophagic proteins and are eventually incorporated into autolysosomes for degradation. We propose that autophagic removal of lysosomes, which we term lysophagy, is a surveillance mechanism that alleviates cells from the adverse effects of lysosomal damage. We envision our method to induce lysosomal damage will enable detailed molecular studies of the lysophagy pathway in the future.

\footnotetext{
${ }^{1}$ Institute of Biological Chemistry, Academia Sinica, Taipei 115, Taiwan. ${ }^{2}$ Institute of Biochemical Sciences, College of Life Sciences, National Taiwan University, Taipei 106, Taiwan. Correspondence and requests for materials should be addressed to W.Y.Y. (email: weiyang@gate.sinica.edu.tw).
} 
ysosomes are ubiquitous cellular organelles responsible for the degradation of endocytosed entities as well as unwanted intracellular materials. The lysosomal degradative actions can be used to generate necessary nutrients from uptaken extracellular food sources, annihilate intruding pathogens and mobilize intracellularly stored materials through autophagy. Because of the large variety of hydrolytic enzymes contained within lysosomal lumens (to carry out the degradation of biomolecules), it was proposed more than 50 years ago by Nobel laureate Christian de Duve that release of lysosomal hydrolases from broken lysosomes into the cell cytoplasm would be detrimental to a cell ${ }^{1}$. Indeed, it is now established that treatment of cells with lysosomotropic agents that destabilize lysosomal membranes in detergent-like fashions cause the release of lysosomal hydrolases such as cathepsins into the cell cytoplasm, leading to apoptosis or necrosis ${ }^{2,3}$. This led us to hypothesize that the existence of lysosome-maintenance mechanisms will alleviate cells from potentially harmful effects of impaired lysosomes.

One potential means to quality control lysosomes is through autophagy. Autophagy, the cellular pathway that targets cellular materials for degradation through the sequestration of cellular materials in double-layered membrane structures termed the phagophores, has already been implicated in the maintenance of cellular organelles such as mitochondria (mitophagy) and peroxisomes (pexophagy) ${ }^{4-8}$. One widely studied organellemaintenance autophagic pathway is parkin-mediated mitophagy ${ }^{8}$. In this pathway, a loss of mitochondrial membrane potential is sensed by the PINK1/parkin machinery, leading to specific ubiquitination of outer-membrane proteins on damaged mitochondria ${ }^{9-11}$. This ubiquitin tag then allows the autophagic machinery and the autophagy adaptor protein p62 to specifically recognize and turnover a dysfunctional mitochondrion within the complex cellular environment ${ }^{10,12-15}$.

In this study, we probe whether autophagy-based maintenance pathways also exist for lysosomes. Through a light-activation scheme, we observe that damaged lysosomes are selectively ubiquitinated, recruite autophagic proteins and are eventually incorporated into autolysosomes for degradation. These observations suggest that autophagy acts as one quality-control mechanism for lysosomes.

\section{Results}

Spatiotemporally controlled induction of lysosomal damage. To probe whether lysosomes can be maintained by autophagy, we established a methodology that enables spatiotemporally controlled impairment of lysosomes within living cells. Lysosome impairment induced by small molecules, such as lysosomotropic agents, results in massive release of lysosomal hydrolases into the cell cytoplasm and triggers cell death responses ${ }^{2,3}$. These treatments inactivate lysosomes globally within a cell (all lysosomes can become targeted) and abrogate the ability of a cell to complete autophagic responses as that requires the presence of active lysosomes. This prompted us to seek alternative strategies in which limited lysosomal damage occurs, minimizing unwanted cell stress and preserving most lysosomes in a cell for possible autophagic clearance mechanisms. To achieve spatiotemporally controlled inactivation of lysosomes, we took advantage of a previously developed strategy for photochemical internalization, a process that facilitates gene delivery through efficient light-induced lysosome breakage ${ }^{16,17}$. In photochemical internalization, a robust photosensitizer is targeted onto lysosomal membranes. Light triggers reactive oxygen species formation at the lysosomal membrane, breaking lysosomes to allow gene delivery into the cell cytoplasm. We utilized disulfonated aluminium phthalocyanine $\left(\mathrm{AlPcS}_{2 \mathrm{a}}\right)$, a robust photosensitizer that targets to lysosomes through endocytosis ${ }^{18}$. Through patterned $635 \mathrm{~nm}$ illumination ${ }^{16}$, $\mathrm{AlPcS}_{2 \mathrm{a}}$ containing lysosomes can be individually impaired with spatiotemporal precision (Fig. 1a). $\mathrm{AlPcS}_{2 \mathrm{a}}$ fluoresces in the far red, making it easy to combine with blue, green and red fluorescent protein constructs for live-cell imaging.

We first confirmed that selective targeting of $\mathrm{AlPcS}_{2 \mathrm{a}}$ onto lysosomes can be achieved in our cultured HeLa cell model. Controlled incubation of $\mathrm{AlPcS}_{2 \mathrm{a}}$ with HeLa cells (Methods) led to the appearance of $\mathrm{AlPcS}_{2 \mathrm{a}}$ in HeLa cells in the form of small cytoplasmic punctate structures resembling endocytic vesicles. a

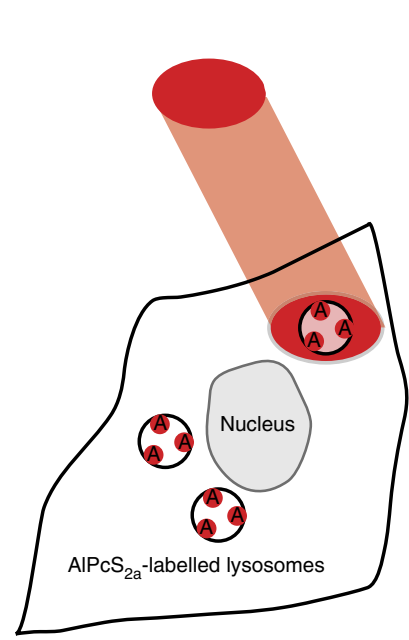

b
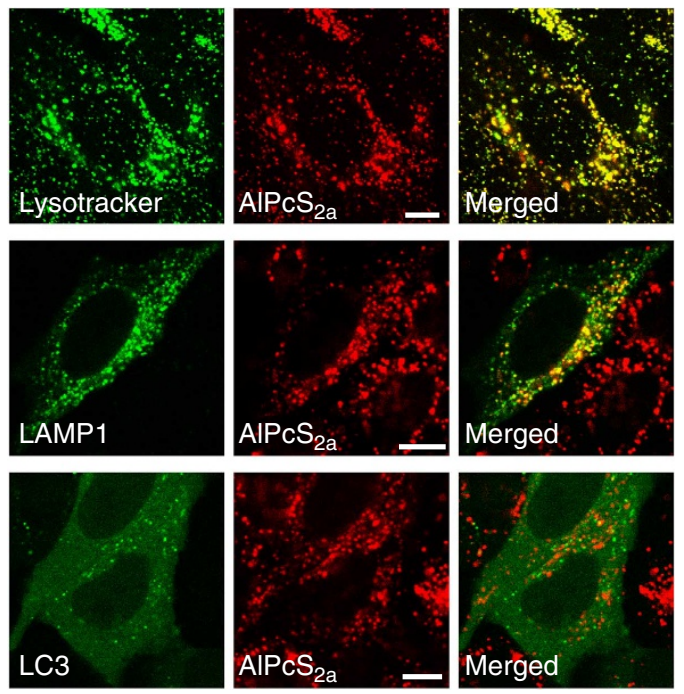

Figure 1 | Targeting AIPcS 2 onto lysosomes for light-activated lysosomal damage. (a) Schematic for spatiotemporally controlled light-activated lysosomal damage. Illumination of individual lysosomes labelled with the robust photosensitizer $\mathrm{AIPcS}_{2 a}$ leads to light-activated damage. (b) AIPcS ${ }_{2 a}$ can be specifically targeted onto lysosomes. Using our staining procedure, AIPcS 2 signals colocalized with Lysotracker Red (top panels) and lamp1-RFP (middle panels), but not with other cellular organelle markers such as EGFP-LC3B (bottom panels, marker for autophagic structures), in HeLa cells. Scale bars, $10 \mu \mathrm{m}$. 
a
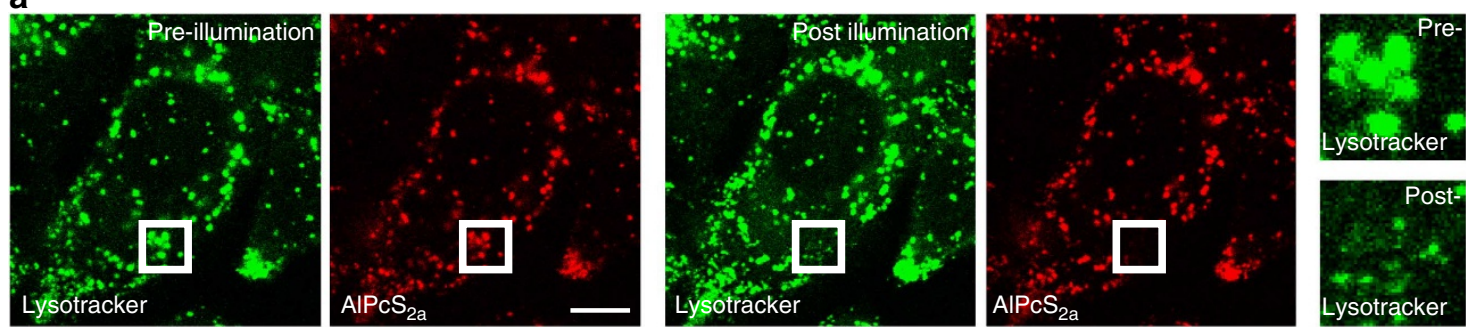

b
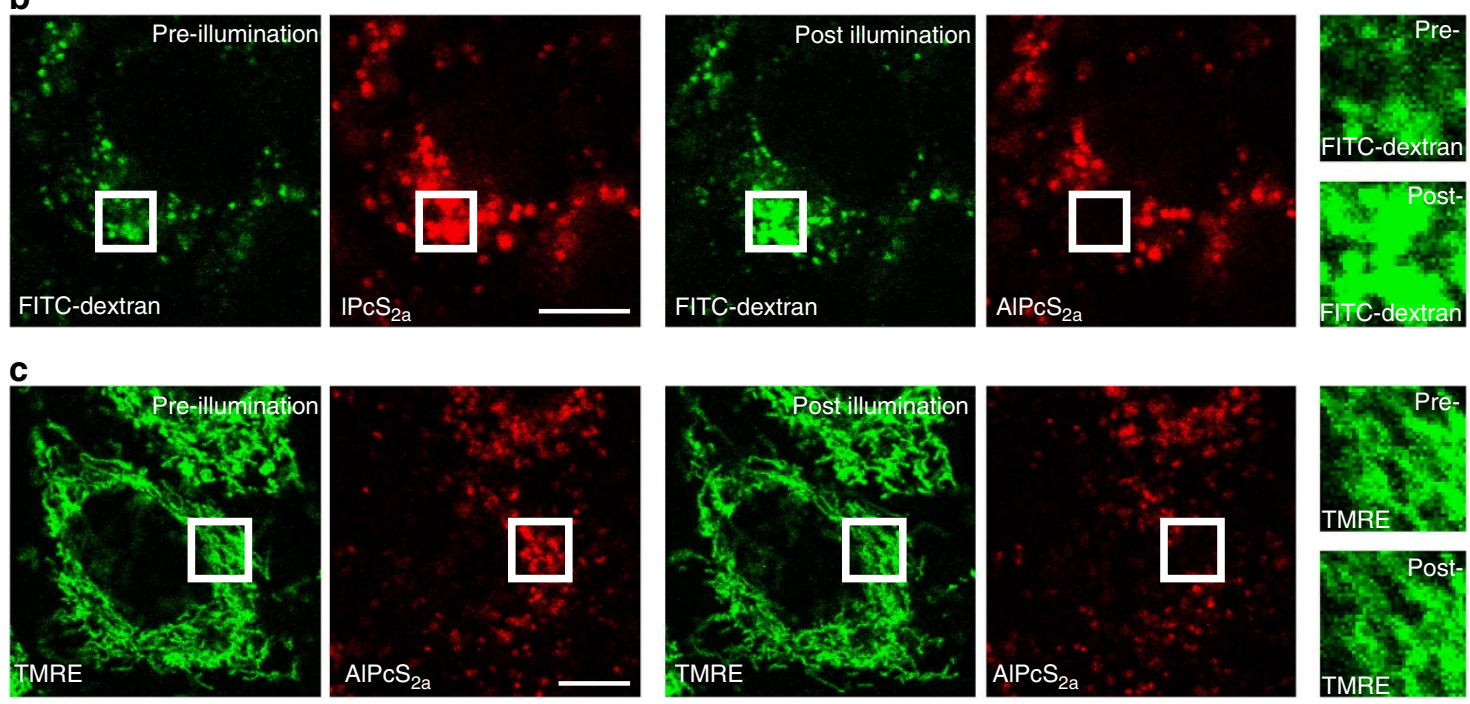

Figure 2 | Induction of LMP on individual AIPcS $\mathbf{2 a}_{\mathbf{2}}$-labelled lysosomes. (a) Simultaneous loss of Lysotracker and AlPcS $_{2 a}$ fluorescence immediately following light activation. A HeLa cell labelled with Lysotracker Red and $\mathrm{AIPCS}_{2 \mathrm{a}}$ was 635-nm illuminated within the white square region (left panels). Lysotracker fluorescence within the illuminated region was immediately lost after light illumination (middle panels), indicating the successful triggering of LMP. Right panels: magnified view of Lysotracker fluorescence within the 635-nm illuminated region pre- and post light activation. (b) $635 \mathrm{~nm}$ illumination did not lead to complete lysosome rupture. A HeLa cell labelled with FITC-dextran (molecular weight $=10,000$ ) and AIPcS $2 a$ was 635-nm illuminated within the white square region (left panels). FITC-dextran remained within illuminated lysosomes (an increase in FITC fluorescence was observed, in accordance with an increase in $\mathrm{pH}$ in damaged lysosomes; middle panels). Right panels: magnified view of FITC-dextran fluorescence within the 635-nm illuminated region pre- and post light activation. (c) Light activation did not affect other cellular structures, such as mitochondria. A HeLa cell labelled with TMRE and $\mathrm{AIPCS}_{2 a}$ was 635-nm illuminated within the white square region (left panels). Illumination did not affect TMRE fluorescence, indicating that mitochondria within the same region were not affected/impaired. Right panels: magnified view of TMRE fluorescence within the 635-nm illuminated region pre- and post light activation. Scale bars, $10 \mu \mathrm{m}$.

Colocalization experiments with lysosomal markers Lysotracker Red or lamp1-RFP (red fluorescent protein) indicated that $\mathrm{AlPcS}_{2 \mathrm{a}}$ was indeed recruited selectively onto lysosomes with our labelling scheme (Fig. 1b). AlPcS ${ }_{2 \mathrm{a}}$ fluorescence, on the other hand, was not found on other organelles, such as peroxisomes, golgi apparatus or mitochondria (Supplementary Fig. S1). AlPcS 2 a fluorescence was also absent on autophagic structures, as evident from the lack of colocalization with enhanced green fluorescent protein (EGFP)-LC3B signals (Fig. 1b).

We next tested the necessary illumination conditions that permit light-mediated lysosomal membrane permeabilization (LMP) in AlPcS $2 \mathrm{z}_{\mathrm{a}}$-stained HeLa cells. The 635-nm illumination pattern, duration and intensity were finely adjusted to achieve the desired degree of lysosomal damage (see Methods). We found that our optimized light treatment can elicit LMP in a spatiotemporally defined fashion within $\mathrm{AlPcS}_{2 \mathrm{a}}$-stained HeLa cells. First, an immediate rise in $\mathrm{pH}$ within selected lysosomes could be achieved following light illumination. In HeLa cells costained with Lysotracker Red and $\mathrm{AlPcS}_{2 \mathrm{a}}, 635-\mathrm{nm}$ illuminated lysosomes immediately lost their AlPcS $2 \mathrm{a}$ fluorescence (due to photobleaching, indicating that the lysosomes were indeed activated by light) as well as their Lysotracker Red signals (due to the rise in $\mathrm{pH}$, Fig. 2a). Second, our light-illumination conditions led to lysosome impairment without inducing full membrane rupture: fluorescein isothiocyanate (FITC)-dextran (molecular weight $=10,000)$ was retained within light-activated lysosomes in $\mathrm{AlPcS}_{2 \mathrm{a}}$-stained HeLa cells (Fig. 2b). Furthermore, the retained FITC-dextran fluorescence greatly increased, again confirming that a rise in $\mathrm{pH}$ occurred within light-activated lysosomes (FITC $\mathrm{pKa}=6.5$, Fig. $2 \mathrm{~b})^{19}$. With highly selective labelling of $\mathrm{AlPcS}_{2 a}$ on the inner membrane of lysosomes, our 635-nm illumination conditions did little damage to other cellular organelles within the same region. Tetramethyl rhodamine ethyl ester perchlorate (TMRE) signals, indicative of active mitochondria (mitochondria are exquisitely sensitive to reactive oxygen species), within the illuminated region remained (Fig. 2c). Similarly, luminal markers for ER and peroxisomes were also unaffected upon light activation of lysosomes (Supplementary Fig. S2). This indicated that the methodology could specifically elicit LMP, and was used to further probe the cellular autophagic response following lysosomal damage. Interestingly, we found that trafficking of photo-impaired lysosomes exhibited reduced motility, allowing them to be easily tracked over long periods of time (Supplementary Fig. S3). 


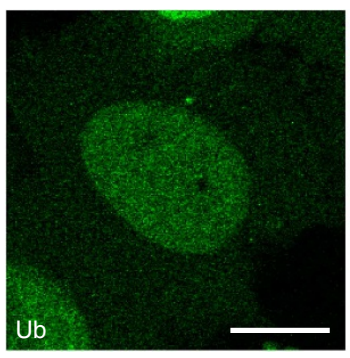

b

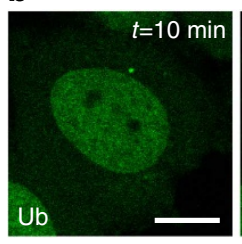

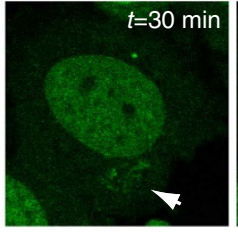

C
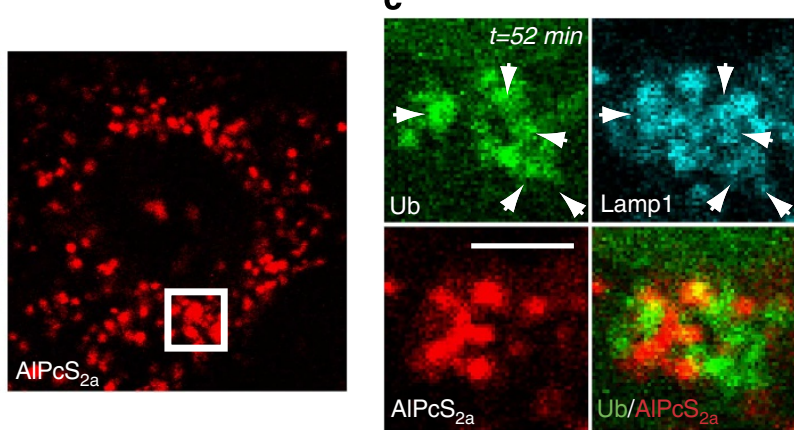

d

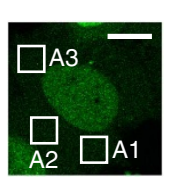

Figure 3 | Ubiquitination of damaged lysosomes. (a) A HeLa cell expressing EGFP-Ub and lamp1-RFP, and labelled with AIPcS $2 a$, was 635-nm illuminated within the white square region. (b) Punctate structures containing ubiquitin appeared within the 635-nm illuminated region (white arrow) 30-50 min after light activation. (c) Ubiquitin puncta colocalized with damaged lysosomes. Magnified view of the 635-nm illuminated cell region showed that ubiquitin signals localized on lysosomes (lamp1-labelled) lacking AIPcS 2 fluorescence (indicating that it had been 635-nm illuminated). White arrows indicate examples of $\mathrm{Ub}$ on damaged lysosomes. (d) Quantification of ubiquitin signals within three independent cellular regions (A1, A2 and A3) revealed a specific recruitment of ubiquitin to the site of light activation (inset, right, A1). Scale bars, $10 \mu \mathrm{m}(\mathbf{a}, \mathbf{b}, \mathbf{d}) ; 3 \mu \mathrm{m}$ (c).

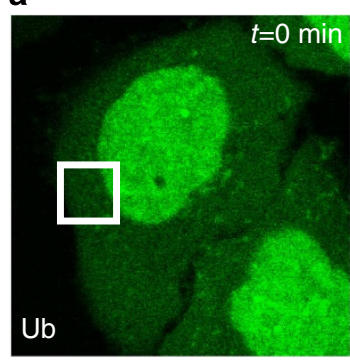

C

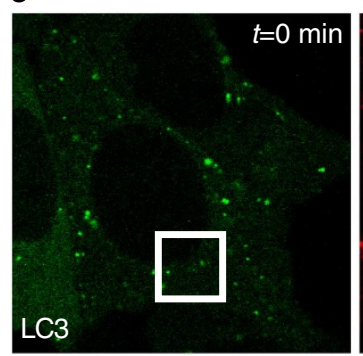

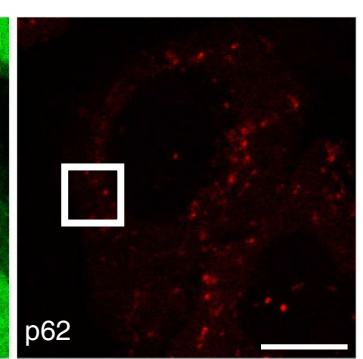

b
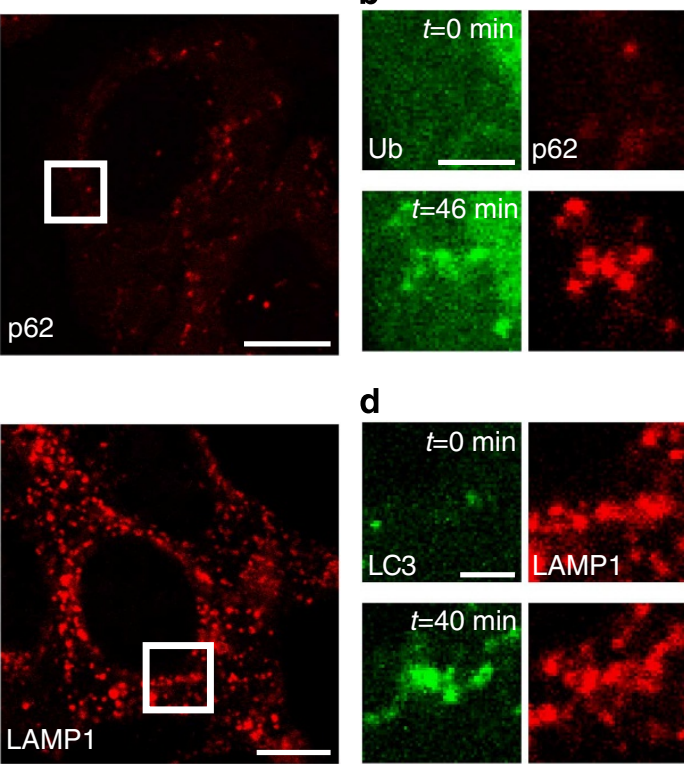

d

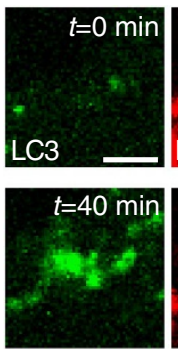

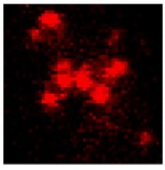
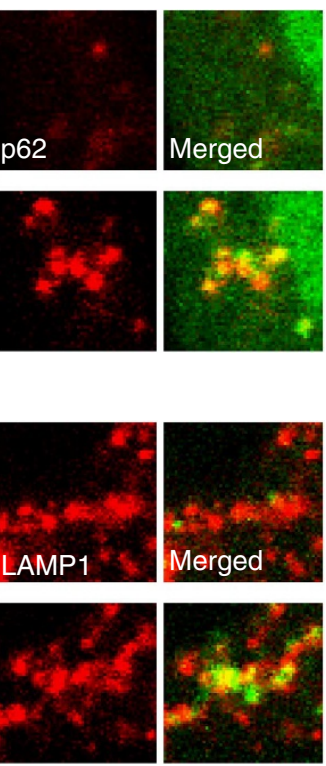

Figure 4 | Specific recruitment of autophagic adaptor proteins onto damaged lysosomes. (a) A HeLa cell expressing EGFP-Ub and TagRFP-p62, and labelled with AlPcS $_{2 a}$, was 635-nm illuminated within the white square region, resulting in ubiquitination of and p62 recruitment to the photodamaged lysosomes, as shown in b. (b) Magnified view of the 635-nm illuminated cell region in a. Ubiquitin and p62 initially distributed uniformly within the region ( $t=0 \mathrm{~min}$, top panels), but formed distinct foci after light activation (bottom panels). (c) A HeLa cell expressing EGFP-LC3B and lamp1-RFP, and labelled with $\mathrm{AIPCS}_{2 a}$, was 635-nm illuminated within the white square region, resulting in LC3 recruitment onto photodamaged lysosomes, as shown in d. (d) Magnified view of the 635-nm illuminated cell region in c. LC3 is initially distributed away from lysosomes (lamp1, $t=0$ min, top panels), but appears on lamp1-positive structures $40 \mathrm{~min}$ after light activation (bottom panels). Scale bars, $10 \mu \mathrm{m}(\mathbf{a}, \mathbf{c}) ; 3 \mu \mathrm{m}(\mathbf{b}, \mathbf{d})$.

Damaged lysosomes undergo selective autophagy. The hallmark for many forms of selective organelle autophagy is surface ubiquitination. In mitophagy, dysfunctional mitochondria are first ubiquitinated before being turned over ${ }^{10,11,13,14,20}$. Artificial ubiquitination of peroxisomal outer-membrane proteins can also lead to pexophagy in mammalian cell cultures ${ }^{21,22}$. We therefore probed whether LMP induction through light activation led to selective ubiquitination of impaired (photo-inactivated) lysosomes within a cell region in an EGFP-ubiquitin (EGFP-Ub), lamp1-RFP expressing $\mathrm{AlPcS}_{2 \mathrm{a}}$-stained HeLa cell (Fig. 3a). Thirty 
a
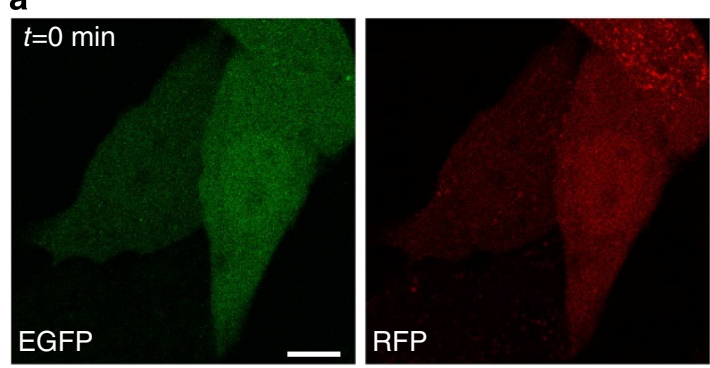

C

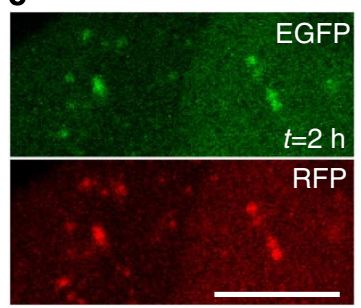

b
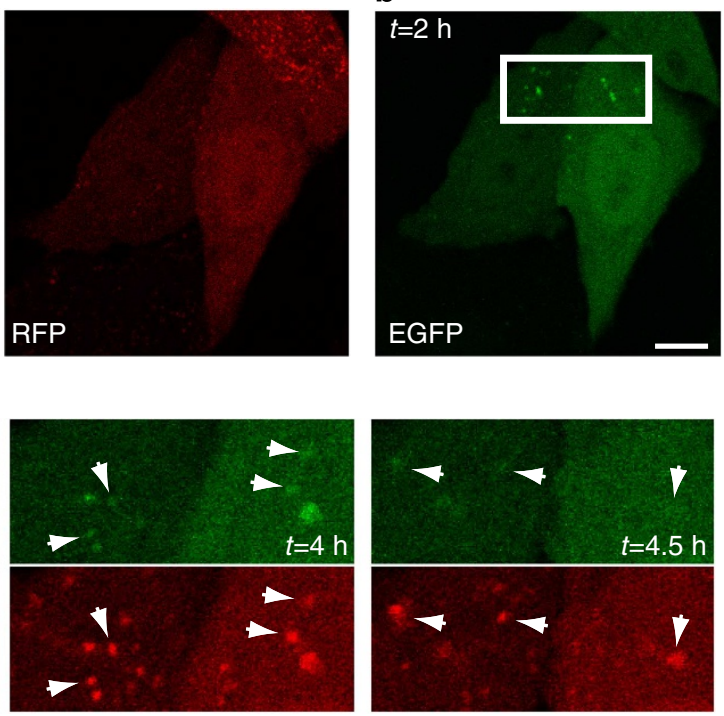

Figure 5 | Delivery of damaged lysosomes for autophagic turnover. (a) Two HeLa cells expressing RFP-EGFP-LC3 and labelled with AIPCS $2 a$ was 635-nm illuminated within the white rectangular region, resulting in local LC3 puncta formation, as shown in b. (b) LC3 puncta were specifically induced within the 635-nm illuminated cell regions in a (LC3 initially distributed uniformly at $t=0$ ). (c) The loss of EGFP fluorescence from light-induced LC3 puncta preceded the disappearance of RFP fluorescence (white arrows indicate examples of LC3 puncta that exhibited diminished EGFP fluorescence), indicating their successful transition from autophagosomes to autolysosomes. Scale bars, $10 \mu \mathrm{m}$.

to fifty minutes after illumination, multiple ubiquitin puncta formed within the $635-\mathrm{nm}$ illuminated region (Fig. 3b), and these EGFP-Ub fluorescence signals were associated with lamp1positive, $\mathrm{AlPcS}_{2 \mathrm{a}}$-fluorescence-negative puncta (Fig. 3c; $\mathrm{AlPcS}_{2 \mathrm{a}}$ is photobleached in light-impaired lysosomes). On the contrary, active lysosomes that were not illuminated by $635 \mathrm{~nm}$ light but were trafficked into the region after illumination were positive for both $\mathrm{AlPcS}_{2 \mathrm{a}}$ and lamp1, and they did not exhibit strong EGFPUb signals, indicating that ubiquitination occurred only on impaired lysosomes. As a control, we quantified three separate regions within the cell for levels of Ub over time, and only the region that has undergone $635 \mathrm{~nm}$ illumination (A1, Fig. 3d) exhibited a time-dependent increase of $\mathrm{Ub}$ signal, again highlighting the selectivity of the process.

We further monitored whether the specific ubiquitination events observed were indicative of autophagic maintenance on damaged lysosomes. This was first tested in $\mathrm{AlPcS}_{2 \mathrm{a}}$-stained HeLa cells expressing TagRFP-p62 and EGFP-Ub (Fig. 4a). p62 is a selective autophagy adaptor protein that is recruited onto substrates during selective autophagy, such as in the turnover of dysfunctional mitochondria or protein aggregates ${ }^{10,23}$. We observed that spatially restricted induction of ubiquitination as well as p62 accumulation occurred in the light-illuminated region (Fig. 4b). p62 and ubiquitin signals were highly associated spatially (Fig. 4b), suggesting ubiquitination as a part of the autophagic response against impaired lysosomes. To further this idea, we probed the cell response to light activation in $\mathrm{AlPcS}_{2 \mathrm{a}^{-}}$ stained HeLa cells expressing EGFP-LC3B and lamp1-RFP (Fig. 4c). Lamp1-RFP signals (lysosomes) within the 635-nm illuminated area were initially not colocalized with EGFP-LC3B, but eventually became decorated with the autophagic structure marker LC3, again implicating a selective autophagic action on impaired lysosomes. Western blotting also revealed an accumulation of cellular LC3-II upon global lysosome impairment (Supplementary Fig. S4a). This accumulation was much more significant than in Bafilomycin A1 (which blocks the turnover of LC3-II) treated cells, confirming that the observed increase in LC3-II levels within light-illuminated cells mainly reflects an induction of autophagy (Supplementary Fig. S4b). Lastly, we demonstrated that our observed autophagic response against lysosome was specific: other forms of selective autophagy, such as parkin-mediated mitophagy, were not induced within the light-illuminated regions (Supplementary Figs S5 and S6).

As only a subset of lysosomes was affected by our method, a cell can maintain normal progression of its autophagic responses (through the remaining healthy lysosome pool). This allowed us to test if the light-damaged lysosomes were successfully turnovered via autophagy using $\mathrm{AlPcS}_{2 \mathrm{a}}$-stained HeLa cells expressing RFP-EGFP-LC3 (ref. 24). We chose two cells that were undergoing very low levels of basal autophagy for imaging (low amounts of RFP or RFP/EGFP-positive autophagic structures before $635 \mathrm{~nm}$ illumination, Fig. 5a). Local formation of LC3 puncta positive for EGFP fluorescence indicated that autophagic processing of light-impaired lysosomes was activated (Fig. 5b). We then tracked whether EGFP fluorescence on these lightinduced autophagic structures disappeared over time: EGFP, with a $\mathrm{pKa}=5.9$, loses its fluorescence when autophagosome transition into autolysosomes occurs ${ }^{24}$. Based on this report, we found that the light-induced autophagic structures indeed successfully matured into autolysosomes (Fig. 5c and Supplementary Fig. S7).

\section{Discussion}

The findings here add to the role of autophagy in organelle maintenance. Organelles are large cellular structures with complex chemical contents that are perhaps best handled through the autophagic machinery (for example, as compared with the proteasome). Previous reports have shown that damaged mitochondria and peroxisomes can be removed via autophagy. Here we report that photo-impaired lysosomes can also be degraded by similar pathways, and the growing list of organelles that undergo autophagic turnover suggests that autophagy can act as versatile organelle quality-control mechanism in cells. On the other hand, it will be of tremendous interest in the future to pinpoint conditions in which physiologically damaged lysosomes need to undergo autophagic turnover. These efforts will further shed light on the utility of this newly defined pathway. 
Aside from being as one of the major organelle quality-control mechanisms, the autophagic response towards impaired lysosomes could act as an intrinsic host-defense mechanism against intruding pathogens. Infection is oftentimes established upon breakage of host cell's vacuolar membranes by pathogens to escape into the cytoplasm ${ }^{25}$. Cellular responses to a break in their vacuolar membrane, such as the autophagic responses reported herein, may allow intruding pathogens to become recaptured for autophagic turnover. Indeed, it has been shown that during Shigella infection, host cell-membrane remnants attached to a bacterium may act as a trigger for autophagy activation ${ }^{26}$. Manipulation of this lysosomemaintenance pathway may therefore represent a useful strategy in combating infectious pathogens.

The light-based lysosome-inactivation methodology developed here allowed us to define the lysosome-maintenance pathway within cells. Our methodology represents a robust experimental platform to access the selective lysosomal autophagic process. Questions such as how damaged lysosomes are sensed, as well as what molecular machineries are required for proper lysosome maintenance, can now be dissected.

\section{Methods}

Plasmids. EBFP2-parkin was described previously ${ }^{27}$. EBFP2-PMP34 and EGFP2p62 were constructed through PCR amplification of human genes from HeLa cell total cDNA, followed by its insertion into the EBFP2-Nuc (Addgene plasmid $14893)^{28}$ and EGFP-C1 vectors. EGFP-vkskl plasmid was constructed through appending the DNA sequence encoding amino acids VKSKL to the EGFP-C1 plasmid. The EGFP-Ub, mRFP-GFP-LC3, lamp1-RFP and EGFP-LC3B plasmids were obtained from Addgene (plasmids 11928, 21074, 1817 and $11546)^{24,29-31}$

Cell culture and transfection. HeLa cells (ATCC, CCL-2) were cultured in DMEM medium (Life Technologies; 11965), supplemented with 10\% FBS (Life Technologies; 10437) and 1\% penicillin/streptomycin (Life Technologies; 15140), and maintained at $37^{\circ} \mathrm{C}$ and $5 \% \mathrm{CO}_{2}$. For transfection into HeLa cells, Lipofectamine 2000 (Life Technologies; 11668) was used based on the manufacturer's protocol. TMRE (Life Technologies; T-669) was supplemented into cells at $100 \mathrm{nM}$ in cell medium for $20 \mathrm{~min}$ at $37^{\circ} \mathrm{C}$. Mitotracker Green (Life Technologies; M7514) and Lysotracker Red (Life Technologies; L7528) were supplemented into cells at $100 \mathrm{nM}$ in cell medium for $1 \mathrm{~h}$ at $37^{\circ} \mathrm{C}$. BODIPY FL C5-ceramide (Life Technologies; D3521) was supplemented into cells at $5 \mu \mathrm{M}$ in cell medium for $30 \mathrm{~min}$ at $4{ }^{\circ} \mathrm{C}$, washed two times with PBS and further chased for $30 \mathrm{~min}$ at $37^{\circ} \mathrm{C}$ before imaging. EGFP-kdel baculoviruses (Life Technologies; C10590) were used at 20 virus particles per HeLa cell for $24 \mathrm{~h}$.

AIPcS 2 labelling. A modified $\mathrm{AlPcS}_{2 \mathrm{a}}$ staining protocol based on previous literature was used ${ }^{16}$. Briefly, a stock solution was prepared by first dissolving $5 \mathrm{mg}$ $\mathrm{AlPcS}_{2 \mathrm{a}}$ (Frontier Scientific; P40632) in $0.2 \mathrm{ml} 0.1 \mathrm{M} \mathrm{NaOH}$, then brought up to a final volume of $1 \mathrm{ml}$ with PBS (final concentration $6.8 \mathrm{mM}$ ). This stock solution was filtered through a $0.2-\mu \mathrm{m}$ filter (Sartorius; 16534) for long-term storage at $-20{ }^{\circ} \mathrm{C}$. To make the staining solution, our $\mathrm{AlPcS}_{2 \mathrm{a}}$ stock was first diluted to $125 \mu \mathrm{M}$ in PBS and further diluted 1,000 folds to a final concentration of $125 \mathrm{nM}$ in DMEM. To stain the lysosomes in HeLa cells, the culturing medium was replaced with the staining solution for $16 \mathrm{~h}$ at $37^{\circ} \mathrm{C}$. The cells were then transferred back into the regular culturing medium for an additional $4 \mathrm{~h}$ at $37^{\circ} \mathrm{C}$ before imaging.

Live-cell manipulation and imaging. Light-assisted autophagy was performed on an Olympus FV1000 confocal microscope $(60 \times$, numerical aperture $=1.2$ water objective or $60 \times$, numerical aperture $=1.3$ silicon oil objective) equipped with a SIM scanner. Before observation, the HeLa cells were transferred into phenol redfree medium for observation (Gibco; 31053, containing FBS and P/S). Live HeLa cells were maintained under $37^{\circ} \mathrm{C}$ and $5 \% \mathrm{CO}_{2}$ on the microscope for manipulation and observation (Tokai Hit; no. MIU-IBC).

To induce LMP with light, we point-scanned a $50-\mu \mathrm{W} 635-\mathrm{nm}$ laser light through a $\sim 7 \times 7-\mu \mathrm{m}^{2}$ region in a HeLa cell for a total of $30 \mathrm{~s}$ using Olympus FV1000's tornado scanning. EBFP2 fluorescence was collected using $405 \mathrm{~nm}$ excitation (collecting 420-480 nm emission). EGFP fluorescence was collected using $488 \mathrm{~nm}$ excitation (collecting $500-550 \mathrm{~nm}$ emission), and TagRFP fluorescence was obtained using $559 \mathrm{~nm}$ excitation (collecting 575-675 nm emission). $\mathrm{AlPcS}_{2 \mathrm{a}}$ fluorescence was collected using $635 \mathrm{~nm}$ excitation (collecting $655-755 \mathrm{~nm}$ emission). A zero-drift compensator module (Olympus) was used to sample drift in the $z$ direction during real-time imaging. The images were not processed after acquisition.
Western blotting. HeLa cells were scraped into RIPA buffer and centrifuged at 15,000 r.p.m. for $10 \mathrm{~min}$. The supernatant was collected and stored at $-20{ }^{\circ} \mathrm{C}$. Samples were separated by electrophoresis (15\% SDS-PAGE), transferred onto polyvinylidene difluoride membranes (Millipore) and probed by anti-LC3B (1:2,000; Novus Biologicals; NB100-2220) and anti-p62 (1:2,000; BD Biosciences; 610832) antibodies.

Lysosome motility assay. We labelled lysosomes in HeLa cells with Lysotracker Blue (Life Technologies; L7525), FITC-dextran (Life Technologies; D1821) and $\mathrm{AlPcS}_{2 \mathrm{a}}$. Intact lysosomes were tracked within single cells through Lysotracker Blue for 30 frames ( $1.5 \mathrm{~s}$ per frame). This was followed by the induction of photodamage on a small fraction of lysosomes through $635 \mathrm{~nm}$ illumination (damaged lysosomes showed increased FITC-dextran fluorescence). The photodamaged lysosomes were then tracked for 30 frames (1.5 s per frame) through FITC-dextran fluorescence. Individual lysosome trajectories were analysed through the particle tracker plugin in Image J.

RFP-GFP-LC3 analysis. GFP/RFP intensity ratios at different time points were analysed by MATLAB. Images were thresholded for autophagic structure identification and calculated for GFP/RFP intensity ratios over time.

\section{References}

1. de Duve, C. in Subcellular Particles. (ed Hayashi, T.) (Ronald Press Co, New York, 1959).

2. Boya, P. \& Kroemer, G. Lysosomal membrane permeabilization in cell death. Oncogene 27, 6434-6451 (2008).

3. Repnik, U., Stoka, V., Turk, V. \& Turk, B. Lysosomes and lysosomal cathepsins in cell death. Biochim. Biophys. Acta. 1824, 22-33 (2012).

4. Kim, I., Rodriguez-Enriquez, S. \& Lemasters, J. J. Selective degradation of mitochondria by mitophagy. Arch. Biochem. Biophys. 462, 245-253 (2007).

5. van Zutphen, T., Veenhuis, M. \& van der Klei, I. J. Damaged peroxisomes are subject to rapid autophagic degradation in the yeast Hansenula polymorpha. Autophagy 7, 863-872 (2011).

6. Narendra, D., Tanaka, A., Suen, D. F. \& Youle, R. J. Parkin is recruited selectively to impaired mitochondria and promotes their autophagy. J. Cell Biol. 183, 795-803 (2008).

7. Till, A., Lakhani, R., Burnett, S. F. \& Subramani, S. Pexophagy: the selective degradation of peroxisomes. Int. J. Cell Biol. 2012, 512721 (2012).

8. Youle, R. J. \& Narendra, D. P. Mechanisms of mitophagy. Nat. Rev. Mol. Cell. Biol. 12, 9-14 (2011)

9. Jin, S. M. et al. Mitochondrial membrane potential regulates PINK1 import and proteolytic destabilization by PARL. J. Cell Biol. 191, 933-942 (2010).

10. Geisler, S. et al. PINK1/Parkin-mediated mitophagy is dependent on VDAC1 and p62/SQSTM1. Nat. Cell. Biol. 12, 119-131 (2010).

11. Narendra, D. P. et al. PINK1 is selectively stabilized on impaired mitochondria to activate Parkin. PLoS Biol. 8, e1000298 (2010).

12. Ding, W. X. et al. Nix is critical to two distinct phases of mitophagy, reactive oxygen species-mediated autophagy induction and Parkin-ubiquitin-p62mediated mitochondrial priming. J. Biol. Chem. 285, 27879-27890 (2010)

13. Okatsu, K. et al. p62/SQSTM1 cooperates with Parkin for perinuclear clustering of depolarized mitochondria. Genes Cells 15, 887-900 (2010).

14. Lee, J. Y., Nagano, Y., Taylor, J. P., Lim, K. L. \& Yao, T. P. Disease-causing mutations in parkin impair mitochondrial ubiquitination, aggregation, and HDAC6-dependent mitophagy. J. Cell Biol. 189, 671-679 (2010).

15. Narendra, D., Kane, L. A., Hauser, D. N., Fearnley, I. M. \& Youle, R. J. p62/ SQSTM1 is required for Parkin-induced mitochondrial clustering but not mitophagy; VDAC1 is dispensable for both. Autophagy 6, 1090-1106 (2010).

16. Prasmickaite, L., Hogset, A. \& Berg, K. Photochemical transfection. Lightinduced, site-directed gene delivery. Methods Mol. Med. 69, 123-135 (2002).

17. Hogset, A. et al. Photochemical internalisation in drug and gene delivery. Adv. Drug Deliv. Rev. 56, 95-115 (2004).

18. Prasmickaite, L., Hogset, A. \& Berg, K. Evaluation of different photosensitizers for use in photochemical gene transfection. Photochem. Photobiol. 73, 388-395 (2001).

19. Marchetti, A., Lelong, E. \& Cosson, P. A measure of endosomal pH by flow cytometry in Dictyostelium. BMC Res. Notes 2, 7 (2009).

20. Matsuda, N. et al. PINK1 stabilized by mitochondrial depolarization recruits Parkin to damaged mitochondria and activates latent Parkin for mitophagy. J. Cell Biol. 189, 211-221 (2010).

21. Kim, P. K., Hailey, D. W., Mullen, R. T. \& Lippincott-Schwartz, J. Ubiquitin signals autophagic degradation of cytosolic proteins and peroxisomes. Proc. Natl Acad. Sci. USA 105, 20567-20574 (2008).

22. Lazarou, M., Jin, S. M., Kane, L. A. \& Youle, R. J. Role of PINK1 binding to the TOM complex and alternate intracellular membranes in recruitment and activation of the E3 ligase Parkin. Dev. Cell 22, 320-333 (2012). 
23. Pankiv, S. et al. p62/SQSTM1 binds directly to Atg8/LC3 to facilitate degradation of ubiquitinated protein aggregates by autophagy. J. Biol. Chem. 282, 24131-24145 (2007).

24. Kimura, S., Noda, T. \& Yoshimori, T. Dissection of the autophagosome maturation process by a novel reporter protein, tandem fluorescent-tagged LC3. Autophagy 3, 452-460 (2007).

25. Goebel, W. \& Kuhn, M. Bacterial replication in the host cell cytosol. Curr. Opin. Microbiol. 3, 49-53 (2000).

26. Dupont, N. et al. Shigella phagocytic vacuolar membrane remnants participate in the cellular response to pathogen invasion and are regulated by autophagy. Cell Host Microbe. 6, 137-149 (2009).

27. Yang, J. Y. \& Yang, W. Y. Spatiotemporally controlled initiation of Parkinmediated mitophagy within single cells. Autophagy 7, 1230-1238 (2011).

28. Ai, H. W., Shaner, N. C., Cheng, Z., Tsien, R. Y. \& Campbell, R. E. Exploration of new chromophore structures leads to the identification of improved blue fluorescent proteins. Biochemistry 46, 5904-5910 (2007).

29. Dantuma, N. P., Groothuis, T. A., Salomons, F. A. \& Neefjes, J. A dynamic ubiquitin equilibrium couples proteasomal activity to chromatin remodeling. J. Cell Biol. 173, 19-26 (2006).

30. Sherer, N. M. et al. Visualization of retroviral replication in living cells reveals budding into multivesicular bodies. Traffic 4, 785-801 (2003).

31. Jackson, W. T. et al. Subversion of cellular autophagosomal machinery by RNA viruses. PLoS Biol. 3, e156 (2005).

\section{Acknowledgements}

This work is supported in part by the AS-99-TP-B09 thematic research grant and a Career Development Award (to W.Y.Y.) from Academia Sinica in Taiwan. We thank Huei-Jiun Yang for her help in carrying out data quantification. We thank Dr Shu Yuan Yang for useful comments regarding the manuscript.

\section{Author contributions}

J.-Y.Y. and W.Y.Y. conceived the light-activation scheme. W.Y.Y. designed the experiments. Y.-H.H. and L.M.-W.C. performed the experiments. W.Y.Y. wrote the paper.

\section{Additional information}

Supplementary Information accompanies this paper at http://www.nature.com/ naturecommunications

Competing financial interests: The authors declare no competing financial interests.

Reprints and permission information is available online at http://npg.nature.com/ reprintsandpermissions/

How to cite this article: Hung, Y.-H. et al. Spatiotemporally controlled induction of autophagy-mediated lysosome turnover. Nat. Commun. 4:2111 doi: 10.1038/ ncomms3111 (2013). 